\title{
BARRIERS TO OBTAINING ANNUAL DIABETIC EYE EXAMS AMONG ADULT RURAL PATIENTS
}

\author{
Gina Koch
}

\section{Gregory Alexander, Dissertation Supervisor}

\begin{abstract}
Purpose: Examine the barriers experienced by adults living in rural areas to obtaining annual diabetic eye exams (DEE) and evaluate the validity and reliability of the Compliance with Annual Diabetic Eye Exams Survey (CADEES). Background: Diabetic retinopathy (DR) is the leading cause of newly diagnosed blindness in adult Americans. Patients should obtain annual (DEE) to diagnosis the earliest stages of DR, when treatment is most effective. Only half of patients currently obtain annual DEE. Theoretical Framework: Health Belief Model (HBM) Methods: Content analysis of the CADEES was based on responses to the open-ended question, "We are interested in why some people do not have yearly eye exams. Can you think of any reasons we did not ask about in this survey?" Construct validity was examined using factor analysis.

Predictive validity was determined using logistic regression, with self-reported adherence as the outcome of interest. Cronbach's alpha was used to evaluate internal consistency as a measure of scale reliability. Differences were evaluated between adherent and non-adherent (to annual DEE) groups, the 45-64 year age and 65+ year groups, and the rural and non-rural groups using two-sample independent t-tests for means. Results: Surveys were mailed to 351 potential participants; 122 respondents were eligible for inclusion (63 females; 59 males). Good strength in validity and reliability were found within CADEES, along with the presence of a strong barriers construct from the HBM. Barriers included "cost" $(p=.003)$, "no treatment available" ( $p=.001)$ and "there are many things that make it hard to get an eye exam every year" $(p<.001)$. Implications: Health care providers must help identify barriers for their patients, to increase adherence to annual DEE.
\end{abstract}

